\title{
Preparation and biological evaluation of a novel pH-sensitive poly ( $\beta$-malic acid) conjugate for antitumor drug delivery
}

\author{
YOUBEI QIAO $^{1 *}$, BAO LIU $^{1 *}$, YIFAN PENG $^{1}$, ERLONG JI ${ }^{2}$ and HONG WU ${ }^{1}$ \\ ${ }^{1}$ Department of Pharmaceutical Analysis, School of Pharmacy, The Fourth Military Medical University, \\ Xi'an, Shaanxi $710032 ;{ }^{2}$ Shiquanhe Medical Station, A'li, Tibet 859000, P.R. China
}

Received April 17, 2018; Accepted September 17, 2018

DOI: 10.3892/ijmm.2018.3893

\begin{abstract}
Poly ( $\beta$-malic acid), referred to as PMLA, has been synthesized and introduced as a polymeric drug carrier due to its desirable biological properties. In the present study, a novel $\mathrm{pH}$-sensitive polymer-drug conjugate based on PMLA, PMLA-Hz-doxorubicin (DOX), was prepared, and another conjugate, PMLA-ami-DOX, was synthesized as a comparison. The structures, conjugation efficiency, and drug release properties of the prodrugs were determined. The cytotoxicity and cell uptake were assessed using the HT1080 human fibrosarcoma cell line as an in vitro cell model. The release of DOX in the two conjugates were pH-dependent in PBS buffer at a $\mathrm{pH}$ of 5.6, 6.0, 6.8 and 7.4. The quantity of drug released increased with the decrease in $\mathrm{pH}$, and PMLA-ami-DOX released twice as much as PMLA-Hz-DOX (12 h). The cytotoxicity of PMLA-Hz-DOX at $\mathrm{pH} 7.4$ was lower than that of free DOX and increased with the decrease in $\mathrm{pH}$, indicating that the cytotoxicity of PMLA-Hz-DOX was $\mathrm{pH}$-sensitive. Flow cytometry and confocal experiments confirmed the efficiency of the PMLA-Hz-DOX conjugate. Therefore, bonding DOX to PMLA via an acid-sensitive hydrazone bond may be used to reduce its toxic side effects on normal tissues while responding to tumor $\mathrm{pH}$ and releasing the drug.
\end{abstract}

\section{Introduction}

Polymeric conjugates of conventional drugs, a conjugation of a drug with a polymer, have several advantages over their low molecular weight precursors. Polymer-drug conjugates can increase the solubility of low solubility or insoluble drugs,

Correspondence to: Professor Hong Wu, Department of Pharmaceutical Analysis, School of Pharmacy, The Fourth Military Medical University, 169 Changle West Road, Xi'an, Shaanxi 710032, P.R. China

E-mail: wuhong@fmmu.edu.cn

${ }^{*}$ Contributed equally

Key words: polymeric drugs, poly ( $\beta$-malic acid), drug delivery, $\mathrm{pH}$-sensitive, doxorubicin enhancing drug bioavailability (1-3). In addition, polymeric conjugates can provide passive and/or active targeting of the drug specifically to the site of its action leading to a reduction in antigenic activity of the drug (4-7). Polymer-drug conjugates have introduced a new era of polymeric drug delivery systems with these advantages over the free form of a drug.

Several polymers with desirable biological properties have been employed as a drug delivery platform. Poly ( $\beta$-malic acid), referred to as PMLA, which is an aliphatic polyester based on malic acid, has a preferable biodegradability and lack of toxicity. PMLA can be degraded into malic acid, an intermediate of tricarboxylic acid cycle, and subsequently degraded into water and $\mathrm{CO}_{2}(8,9)$. PMLA has free pendant carboxyl groups on each monomer, which can be covalently bound to small-molecule drugs and other functional moieties (10-12). Multi-modification makes PMLA an efficient carrier, making it well-suited as a scaffold for tailored nanoconjugate chemistry.

Polymeric-drug conjugates based on PMLA have been examined over the course of the last two decades (13). Ljubimova et al (14) synthesized a targeted polymeric delivery system based on PMLA, named Polycefin, which is constructed of antisense oligonucleotides targeting Laminin-8 and monoclonal anti-transferrin receptor antibody, to target brain tumors and breast cancer. Polycefin was found to accumulate in U87MG brain tumor tissue and had no toxic effects on normal tissue (14-16). Controlled molecular weight PMLA was synthesized with a high yield in our previous study, and the antitumor agent 10-hydroxycamptothecin was attached to PMLA in order to enhance its water solubility and bioavailability $(17,18)$.

To further improve delivery efficiency and cancer-targeting specificity, environment-responsive nanoconjugates have been developed by employing external stimuli, including $\mathrm{pH}$-responsiveness. The application of $\mathrm{pH}$-sensitivity is based on the fact that increased aerobic glycolysis in cancer cells leads to a lower extracellular $\mathrm{pH}$ of cancer cells $(\mathrm{pH}$ 6.5-7.2) than that in normal tissues $(19,20)$. In addition, once taken up by cells, drug carriers experience a gradient $\mathrm{pH}$ moving from endosomes ( $\mathrm{pH}$ 5.0-6.0) to lysosomes ( $\mathrm{pH} 4.0-4.5)$ (21-23). One approach is to introduce cleavable bonds, which can be broken to release the drugs conjugated to or encapsulated in the carrier (24-26). In the case of polymer-drug conjugates, $\mathrm{pH}$-sensitive linkages, including hydrazone, hydrazide and 
acetal linkages, have been used to directly attach drug molecules to polymers $(19,27-29)$.

In the case of polymer-drug conjugates, $\mathrm{pH}$-sensitive linkages, including hydrazone, hydrazide, and acetal linkages, have been used to directly attach drug molecules to polymers (24). Based on our previous study, a pH-triggered drug release profile based on PMLA, PMLA-Hz-doxorubicin (DOX), was examined in the present study. The anticancer drug DOX was chemically attached to the polymer backbone via a $\mathrm{pH}$-responsive hydrazone bond. By contrast, another conjugate linking DOX via an amide bond was also prepared. The polymeric conjugation protected DOX from being released during systemic circulation, however, once the polymeric drug was internalized by the cancer cells and taken up by the endo/lysosomes, the hydrazone linkage was ruptured due to the acidic microenvironment and DOX was released from the PMLA backbone (Fig. 1). It is anticipated that the $\mathrm{pH}$-triggered release of the nanoconjugate may enhance the drug delivery efficiency and offer a promising therapeutic outcome.

\section{Materials and methods}

Materials. L-aspartic acid and trifluoroacetic anhydride were obtained from Aladdin Chemical Co., Ltd. (Shanghai, China) Doxorubicin hydrochloride (DOX $\mathrm{HCl}$ ) was purchased from HVSF United (Beijing, China). 1-(3-dimethylaminopropyl)-3-ethylcarbodiimide hydrochloride (EDC $\cdot \mathrm{HCl})$ and $\mathrm{N}$-hydroxysuccinimide (NHS) were purchased from TCI (Shanghai, China). HPLC-grade acetonitrile and methanol were purchased from Merck KGaA (Darmstadt, Germany). Minimum Eagle's medium (MEM) and trypsin were purchased from HyClone; GE Healthcare Life Sciences (Logan, UT, USA) and Gibco; EMD Millipore (Billerica, MA, USA), respectively. The Cell Counting Kit-8, fetal bovine serum (FBS) and 4',6-diamidino-2-phenylindole (DAPI) were purchased from ZETA life (Shanghai, China). All other chemicals used in the present study were obtained from Sinopharm Chemical Reagent Co., Ltd. (Shanghai, China), and all reagents were of analytical grade without further purification.

Synthesis of PMLA. PMLA was synthesized as described previously by ring-opening polymerization, starting from L-aspartic acid $(17,18)$. PMLA with a molecular weight of $10 \mathrm{kDa}$ was used in the present study, which is a suitable molecular weight for drug conjugates. The yield of PMLA was 5.6\%. The synthetic route for PMLA is illustrated in Fig. 2.

Preparation of PMLA-Hz-DOX and PMLA-ami-DOX. PMLA (116 $\mathrm{mg}, 1 \mathrm{mmol}$ with regard to repeat units), NHS (1 mmol) and $\mathrm{EDC} \cdot \mathrm{HCl}(1.5 \mathrm{smmol})$ were dissolved in $15 \mathrm{ml}$ of DMSO and stirred at room temperature for $6 \mathrm{~h}$ to activate the carboxyl group. Subsequently, $2 \mathrm{mmol} \mathrm{N}_{2} \mathrm{H}_{4} \cdot \mathrm{H} 2 \mathrm{O}$ was added. After $6 \mathrm{~h}, 0.2 \mathrm{mmol}$ DOX was added to the solution and stirred at room temperature for another $6 \mathrm{~h}$ in the dark. The solution was dialyzed (MWCO: $5 \mathrm{kDa}$ ) against DMSO for $24 \mathrm{~h}$ to remove unreacted and produced small molecules and then dialyzed (MWCO: $5 \mathrm{kDa}$ ) against deionized water at $4^{\circ} \mathrm{C}$ in the dark for $24 \mathrm{~h}$. Finally, the solution was lyophilized to yield PMLA-Hz-DOX as red powder, the yield was $64.5 \%$.
The carboxyl group was activated, as above, and then $0.2 \mathrm{mmol}$ DOX was added and stirred at room temperature overnight in the dark. The solution was dialyzed (MWCO: $5 \mathrm{kDa}$ ) against DMSO for $24 \mathrm{~h}$ to remove unreacted regent and produced small molecules and then dialyzed (MWCO: $5 \mathrm{kDa}$ ) against deionized water at $4^{\circ} \mathrm{C}$ in the dark for $24 \mathrm{~h}$. Finally, the solution was lyophilized to yield PMLA-ami-DOX as red powder with a yield of $76.6 \%$. The synthetic routes for these two conjugates are illustrated in Fig. 3.

UV-vis spectroscopy (Shimadzu Corporation, Tokyo, Japan) was used to quantify the quantity of DOX conjugated on the polymers. Briefly, the DOX-conjugated polymers were dissolved in deionized water and the absorbance of the solutions at $254 \mathrm{~nm}$ was measured. Using a calibration curve obtained by measuring the absorbance of different concentrations of free DOX in deionized water at $254 \mathrm{~nm}$, the DOX content in the polymer was calculated. The DOX loading rate was calculated follows: DOX loading rate $(\%)=($ weight of DOX in conjugate/weight of conjugate) $\mathrm{x} 100 \%$; and the conjugating rate was calculated as follows: Conjugating rate $(\%)=($ weight of DOX in conjugate/weight of DOX input) $x 100 \%$.

Characterization of PMLA-Hz-DOX and PMLA-ami-DOX. The chemical structures of the synthesized PMLA-Hz-DOX and PMLA-ami-DOX were determined by FT-IR using a $\mathrm{KBr}$ disc (Shimadzu FTIR-8400S) and ${ }^{1} \mathrm{H}$ NMR using Varian $400 \mathrm{mHz}$ spectrometer (Avance, Bruker Corporation, Billerica, MA, USA). The samples were prepared from freeze-dried products by dissolving in DMSO-d6 $(20 \mathrm{mg} / \mathrm{ml})$ at $25^{\circ} \mathrm{C}$. Chemical shifts were determined in $\delta$ units relative to the tetramethyl silane signal as an internal reference.

The $\zeta$ potential of these two conjugates was determined by dynamic light scattering (DLS) following dissolving in water.

In vitro release of DOX from the conjugates. The in vitro drug release profiles were obtained by a dynamic dialysis method (30). The release experiments were performed at $37^{\circ} \mathrm{C}$. Typically, the conjugate solution of $200 \mu \mathrm{g} / \mathrm{ml}$ equivalent DOX concentration was dialyzed into $100 \mathrm{ml}$ of $0.1 \mathrm{M}$ phosphate-buffered saline (PBS; pH 5.6, 6.0, 6.8, and 7.4) with magnetic stirring at $200 \mathrm{rpm}$. At hourly intervals, $0.5 \mathrm{ml}$ was removed from the release medium for each sample, and the same volume and temperature of PBS was added to the release medium. The released DOX was determined by HPLC (Agilent 1260), according to Chinese Pharmacopoeia 2015 (ChP 2015) (31). The results of the triplicate tests were used to calculate the accumulated drug release.

In vitro cytotoxicity. HT1080 fibrosarcoma cells, which were provided by Shanghai Zhongqiaoxinzhou Biotechnology Co., Ltd. (Shanghai, China), were used as in vitro models. The cells were cultured in MEM containing 10\% heat-activated FBS and $100 \mathrm{IU} / \mathrm{ml}$ penicillin and $100 \mu \mathrm{g} / \mathrm{ml}$ streptomycin. They were incubated in a $37^{\circ} \mathrm{C}$ water-jacketed incubator equilibrated with $5 \% \mathrm{CO}_{2}$ and maintained at $\sim 99 \%$ relative humidity. The medium was replenished every other day until confluence was achieved. The cells were then washed with PBS and harvested with $0.125 \%$ trypsin-EDTA solution.

The HT1080 fibrosarcoma cells were seeded at a density of $5 \times 10^{4}$ cells/well in a 96-well transparent plate and incubated 




Figure 1. Schematic illustration of tumor cell uptake of nanoconjugates and pH-triggered drug release. PMLA, poly ( $\beta$-malic acid); DOX, doxorubicin.

for $24 \mathrm{~h}$. All growth medium was prepared by supplementing MEM with $10 \%$ FBS and sterilized with a $0.2 \mu \mathrm{m}$ filter prior to use. The medium was then replaced with PMLA-Hz-DOX, PMLA-ami-DOX or free DOX at various drug concentrations $(0.005,0.01,0.05,0.1,0.5$ and $1 \mu \mathrm{g} / \mathrm{ml})$ in medium at $\mathrm{pH} 6.0$ or 7.4. The cells were then incubated for another $48 \mathrm{~h}$ prior to replacing the medium with $01 \mathrm{ml}$ of fresh growth medium containing 10\% CCK-8. Following incubation for another $2 \mathrm{~h}$, the plates were vigorously shaken prior to measuring the relative color intensity at $450 \mathrm{~nm}$ using a microplate reader. Cell viability was determined as a percentage of the intensity of the controls \pm standard deviation. Each experiment was repeated five times at each polymer concentration.

The cell viability with PMLA alone was also investigated at various concentrations equivalent to those for the conjugate.

Confocal laser scanning microscopy (CLSM) observation. The HT1080 cells were maintained in a 6-well plate at $5 \times 10^{5}$ cells/well for $24 \mathrm{~h}$ and treated with PMLA-Hz-DOX, PMLA-ami-DOX or free DOX for $2 \mathrm{~h}$ at $37^{\circ} \mathrm{C}$. Following incubation, the cells were washed with PBS three times to remove excess conjugates. The concentration of DOX was $5 \mu \mathrm{g} / \mathrm{ml}$. The cells were the washed with PBS three times, fixed in precooled $4 \%$ paraformaldehyde for $20 \mathrm{~min}$, and stained with DAPI nuclear stain $(5 \mu \mathrm{g} / \mathrm{ml})$ for $5 \mathrm{~min}$ at $4^{\circ} \mathrm{C}$ in the dark. The cells were washed with PBS, and fluorescent images of cells were analyzed using an FV1000 confocal microscope (Olympus Corporation, Tokyo, Japan).

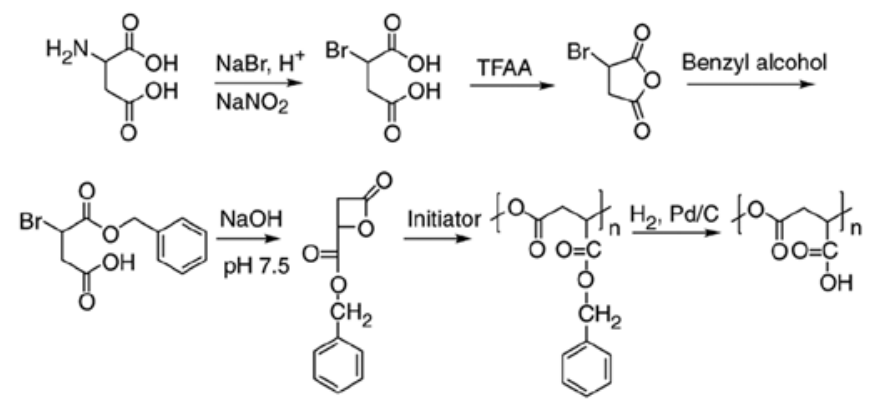

Figure 2. Synthesis of poly ( $\beta$-malic acid).

Flow cytometric analysis. As described in detail previously (32), flow cytometry was used to confirm the uptake of conjugates by HT1080 cells. Similar to the confocal study, the HT1080 cells were seeded in a 6 -well plate at $5 \times 10^{5}$ cells/well for $24 \mathrm{~h}$ and treated with PMLA-Hz-DOX, PMLA-ami-DOX or free DOX for $2 \mathrm{~h}$ at $37^{\circ} \mathrm{C}$. Following incubation, the cells were washed with PBS three times. The cells were then harvested by trypsinization, centrifuged at $352 \mathrm{x}$ g for $5 \mathrm{~min}$, at $4^{\circ} \mathrm{C}$, resuspended in $500 \mu \mathrm{l}$ PBS medium and examined by flow cytometry using a FACScan instrument (BD Biosciences, Franklin Lakes, NJ, USA).

Statistical analysis. All experiments were performed in triplicate and the obtained data were processed using GraphPad 
<smiles>CC(C)(CC(=O)OC(CC(=O)OC(CC(=O)OC(CC(=O)O)C(=O)O)C(=O)O)C(=O)O)C(=O)O</smiles><smiles>CC(C)(C)C(CC(=O)OC(CC(=O)OC(CC(=O)OC(CC(=O)O)C(=O)OC1C(=O)CCC1=O)C(=O)OC1C(=O)CCC1=O)C(=O)OC1C(=O)CCC1=O)C(=O)O</smiles>

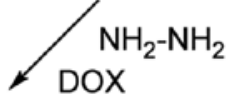

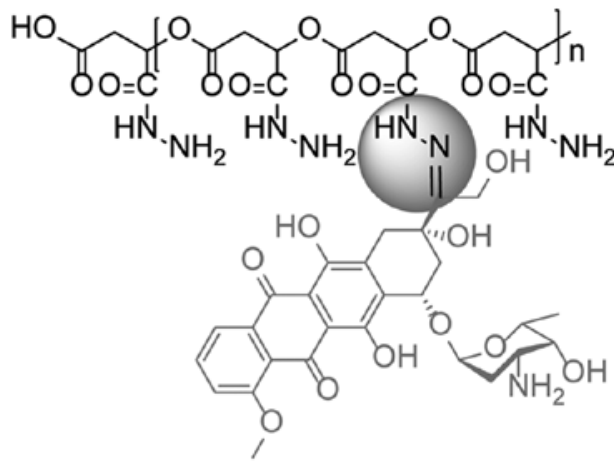

PMLA-Hz-DOX

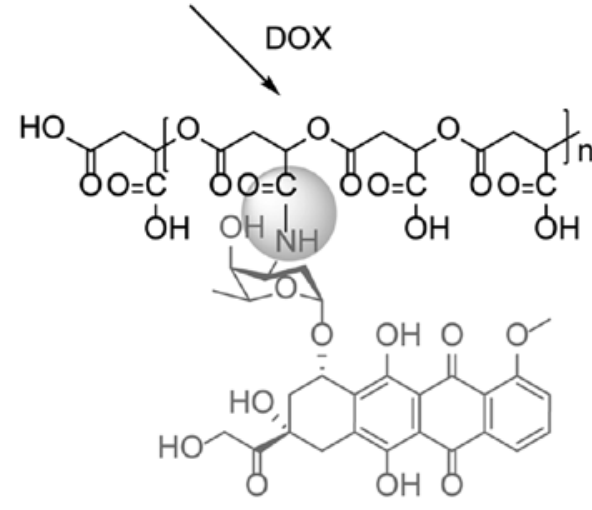

PMLA-ami-DOX

Figure 3. Synthesis of PMLA-Hz-DOX and PMLA-ami-DOX. PMLA, poly ( $\beta$-malic acid); DOX, doxorubicin.

Prism 7 (GraphPad Software, Inc., La Jolla, CA, USA) and Origin 2018 (OriginLab, Northampton, MA, USA) software. Differences between the two groups were analyzed using the unpaired t-test in these software programs. $\mathrm{P}<0.05$ was considered to indicate a statistically significant difference.

\section{Results and Discussion}

Drug loading and conjugation rate of PMLA-Hz-DOX and PMLA-ami-DOX. The DOX loading rates of the PMLA-Hz-DOX and PMLA-ami-DOX conjugates were $20.09 \pm 2.64$ and $19.13 \pm 3.30 \mathrm{wt} \%$, respectively, and the DOX conjugating rates were $69.28 \pm 9.11$ and $65.96 \pm 11.39 \%$, respectively. A large number of suspended carboxyl groups on the PMLA backbone makes it a high drug loading carrier compared with other polymers, including PEG, PLA, PCL and other polyesters that have no active groups on the main chains, meaning drugs may only be encapsulated with a loading rate of $<5 \%(1,2)$.

Characterization of PMLA-Hz-DOX and PMLA-ami-DOX. In PMLA-Hz-DOX, the carbonyl group at the $\mathrm{C}-13$ position of DOX reacts with the hydrazide of the PMLA side chain to form a hydrazone bond whereas the amino group at the 3 position of the DOX pyran ring in PMLA-ami-DOX forms an amide bond with the carboxyl group on PMLA. ${ }^{1} \mathrm{H}$ NMR

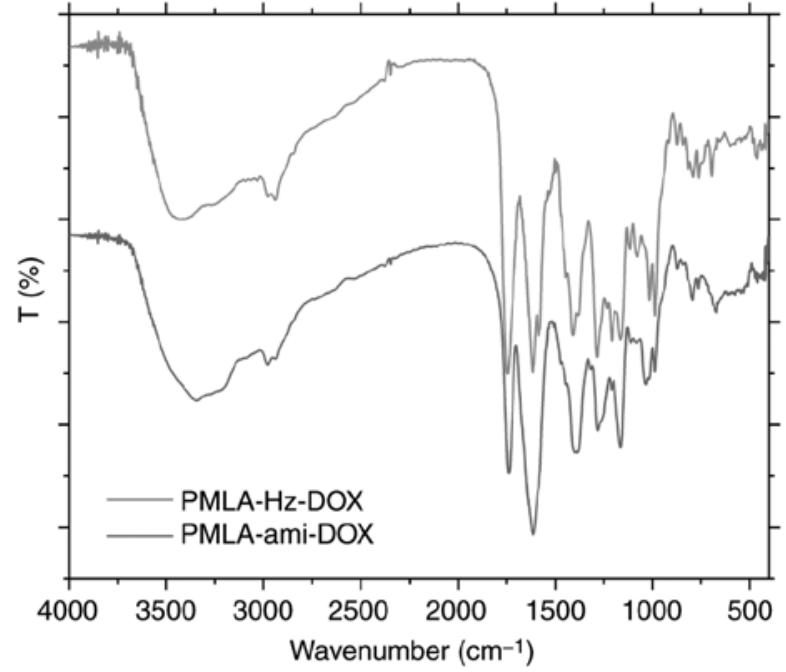

Figure 4. IR spectra of PMLA-Hz-DOX and PMLA-ami-DOX. PMLA, poly ( $\beta$-malic acid); DOX, doxorubicin; $T$, transmittance.

in DMSO- $\mathrm{d}_{6}$ was used to confirm the product identity. The IR spectra of these two conjugates are shown in Fig. 4. IR $\left(v, \mathrm{~cm}^{-1}\right): 1,585\left(-\mathrm{NH}_{2}, \delta\right), 1,381(\mathrm{C}-\mathrm{N}, \mathrm{st})$. The peak close to $1,540 \mathrm{~cm}^{-1}$ in the IR spectrum of PMLA-Hz-DOX was $\delta_{\mathrm{NH}}$ (-CO-NH-). The ${ }^{1} \mathrm{H}-\mathrm{NMR}$ spectrum of PMLA-Hz-DOX and 

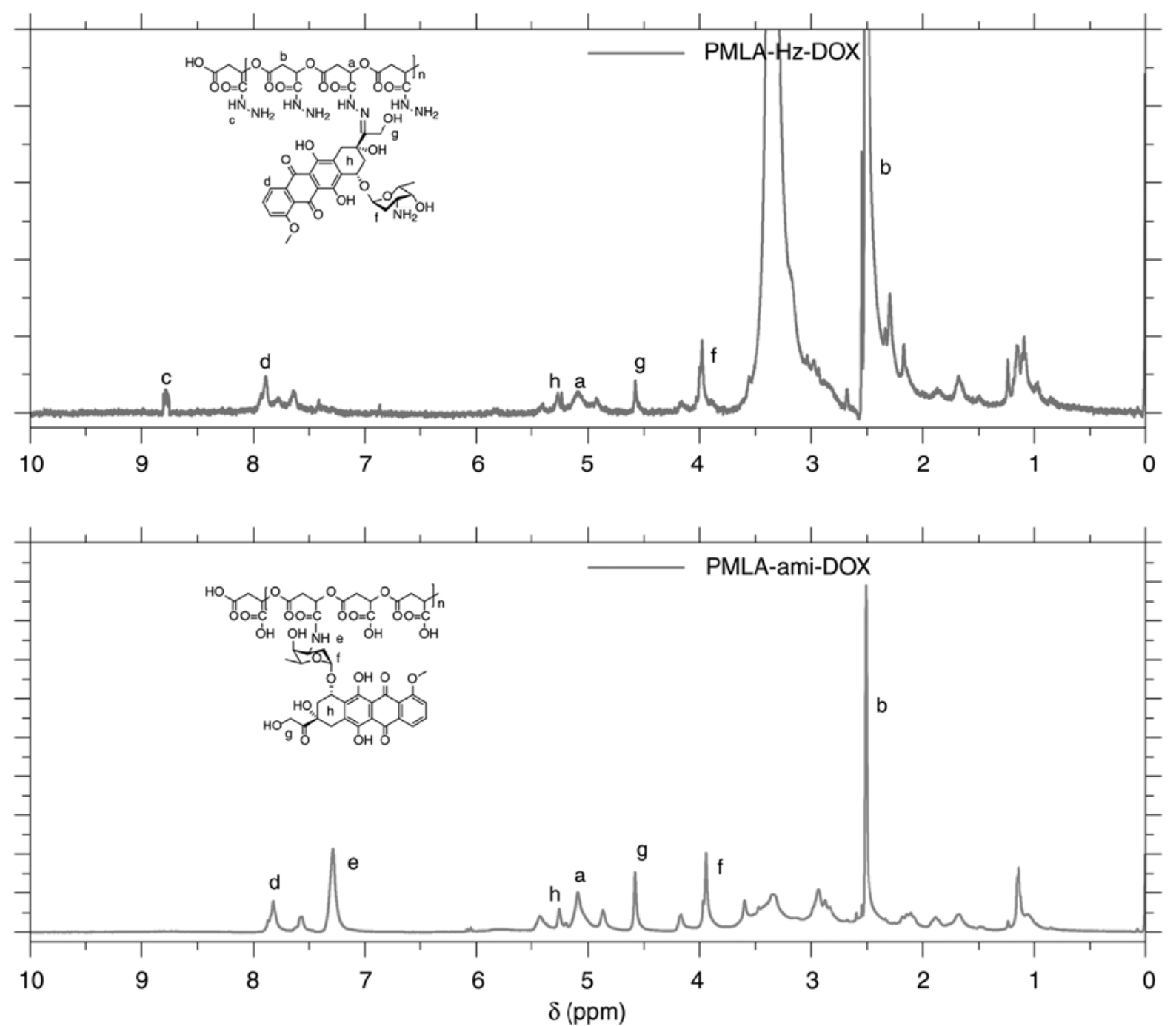

Figure 5. ${ }^{1} \mathrm{H}-\mathrm{NMR}$ of PMLA-Hz-DOX and PMLA-ami-DOX. PMLA, poly ( $\beta$-malic acid); DOX, doxorubicin. a-h, position of protons on DOX.

PMLA-ami-DOX structures are shown in Fig. 5, in which the peaks have been identified and marked. The peaks at different positions of a-h correspond to different protons on DOX.

The $\zeta$ potential of these two conjugates was determined by DLS (Table I). PMLA without the DOX connection had negative $\zeta$ potential and, once DOX was conjugated, it showed positive $\zeta$ potential. The $\zeta$ potential of PMLA-Hz-DOX was $20.25 \pm 0.36 \mathrm{mV}$ due to the modification of the amino group on the carboxyl group and the introduction of DOX, whereas the $\zeta$ potential of PMLA-ami-DOX was $10.57 \pm 0.42 \mathrm{mV}$. The positively charged nanoconjugates effectively interact with the negatively charged cell membrane by electrostatic attraction, triggering efficient cell internalization $(33,34)$.

In vitro release of DOX from the conjugates. The in vitro release of the drug from PMLA-Hz-DOX and PMLA-ami-DOX conjugates was measured at various $\mathrm{pH}$ conditions. As shown in Fig. 6, the DOX release showed no marked initial burst in PMLA-Hz-DOX. It was, however, significantly $\mathrm{pH}$-dependent; the lower the $\mathrm{pH}$ was, the faster the drug released. Specifically, the drug released rapidly from the PMLA-Hz-DOX conjugate at pH 5.6 and pH 6.0, reaching
Table I. $\zeta$ potential of PMLA-ami-DOX and PMLA-Hz-DOX.

\begin{tabular}{lc}
\hline Conjugate & $\zeta$ potential $(\mathrm{mV})$ \\
\hline PMLA-Hz-DOX & $20.25 \pm 0.36$ \\
PMLA-ami-DOX & $10.57 \pm 0.42$
\end{tabular}

DOX, doxorubicin; PMLA, poly ( $\beta$-malic acid).

70.0 and $54.9 \%$, respectively, at $24 \mathrm{~h}$, whereas DOX release at pH 6.8 and pH 7.4 was markedly slower at 16.87 and $9.93 \%$ in the same period, respectively. For PMLA-ami-DOX, DOX release was slower than that of PMLA-Hz-DOX, and there was no $\mathrm{pH}$ dependence. Using this polymeric drug design, the conjugates can stably preserve drugs under physiological conditions and selectively degrade and release them by responding to the tumor extracellular $\mathrm{pH}\left(\mathrm{pH}_{\mathrm{e}}\right)$, endosomes ( $\mathrm{pH} 5-6)$ or lysosomes $(\mathrm{pH} 4-5)(25,35,36)$. The in vitro drug release experiments showed that the release of DOX was $\mathrm{pH}$-dependent. When the conjugate reached the tumor tissue, DOX was released, and the free and grafted DOX entered the 

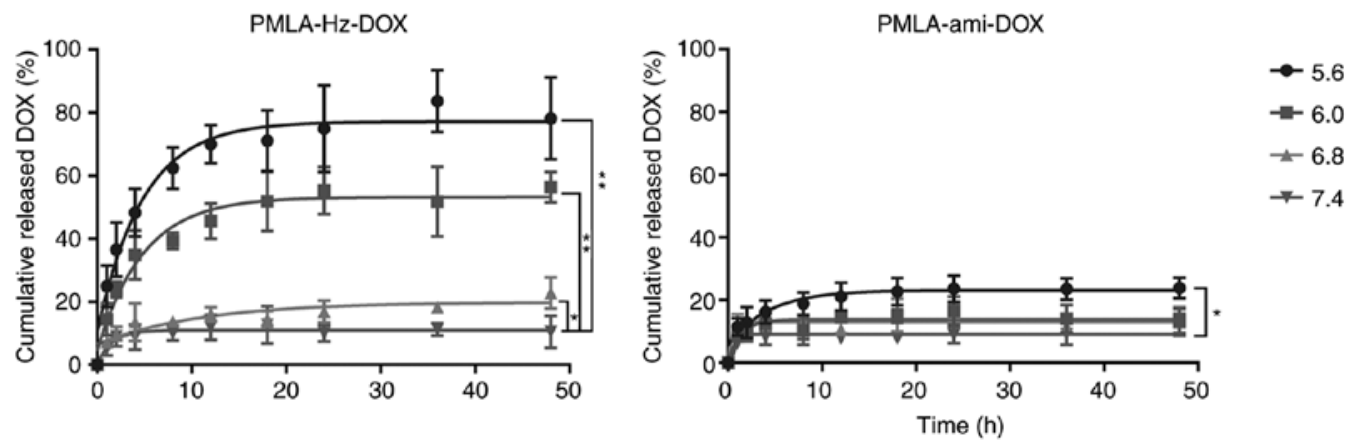

Figure 6. Cumulative release profile of PMLA-Hz-DOX and PMLA-ami-DOX at different $\mathrm{pH}$ values at $37^{\circ} \mathrm{C}$. PMLA, poly ( $\beta$-malic acid); DOX, doxorubicin. ${ }^{* *} \mathrm{P}<0.01$ and ${ }^{*} \mathrm{P}<0.05$ vs. $\mathrm{pH}$ 7.4.

tumor cells by diffusion or endocytosis (Fig. 1). In the tumor cell endosome, in which $\mathrm{pH}$ was as low as 4-5, DOX release was increased, although the release rate of DOX in tumor tissues requires further improvement.

In vitro cytotoxicity. The in vitro antitumor activities of various DOX formulations were evaluated using a CCK-8 assay of HT1080 fibrosarcoma cells, and the 50\% inhibitory concentration $\left(\mathrm{IC}_{50}\right)$ was determined. PMLA did not show cytotoxicity up to a concentration of $1 \mathrm{mg} / \mathrm{ml}$, making it safe to use as a drug carrier (Fig. 7). The in vitro cytotoxicity at different $\mathrm{pH}$ values exhibited $\mathrm{pH}$-dependent cytotoxic effects (Fig. 8). At pH 7.4, the two conjugates showed lower $\mathrm{IC}_{50}$ values than free DOX, suggesting that DOX grafted to PMLA is likely to have lower side effects in systemic circulation. At $\mathrm{pH}$ 6.0, the $\mathrm{pH}$ of cell endosomes, PMLA-Hz-DOX had a higher cell cytotoxicity $\left(\mathrm{IC}_{50}=0.026 \mu \mathrm{g} / \mathrm{ml}\right)$; however, the cell viability of PMLA-ami-DOX was markedly higher $\left(\mathrm{IC}_{50}=0.31 \mu \mathrm{g} / \mathrm{ml}\right)(\mathrm{P}<0.05)$. According to the structure-activity association of DOX, the carbonyl of C-13 may interact with the DNA double helix by hydrogen bond, which is crucial for maintaining its activity. Following linking to PMLA via a hydrazone bond, the antitumor activity reduced. These results suggested that the cytotoxicity of polymeric conjugates against HT1080 cells was lower than free DOX under physiological $\mathrm{pH}$.

Cellular uptake measured by CLSM and flow cytometry. CLSM and flow cytometry were performed to investigate the effects of $\mathrm{pH}$-sensitivity on the cellular uptake of nanoconjugates. As shown in Fig. 9, the PMLA-ami-DOX conjugate showed the weakest intracellular fluorescence of DOX at $\mathrm{pH} 7.4$ and 6.0, indicating the DOX grafted to PMLA via an amide bond had reduced cell uptake. Compared with PMLA-ami-DOX, PMLA-Hz-DOX showed higher intracellular fluorescence of DOX at $\mathrm{pH} 6.0$ than that at $\mathrm{pH} 7.4$, suggesting that PMLA-Hz-DOX was more efficient following uptake by cells.

Flow cytometry was used to further examine the cellular uptake of the DOX-loaded nanoconjugates. As shown in Fig. 10, following incubation with HT1080 cells for $2 \mathrm{~h}$, the two nanoconjugates showed relatively low cell internalization at $\mathrm{pH}$ 7.4. When the $\mathrm{pH}$ decreased to 6.0, PMLA-ami-DOX showed similar cellular uptake, however, the cellular uptake of PMLA-Hz-DOX was increased $(\mathrm{P}<0.05)$. These results were consistent with the CLSM analysis.

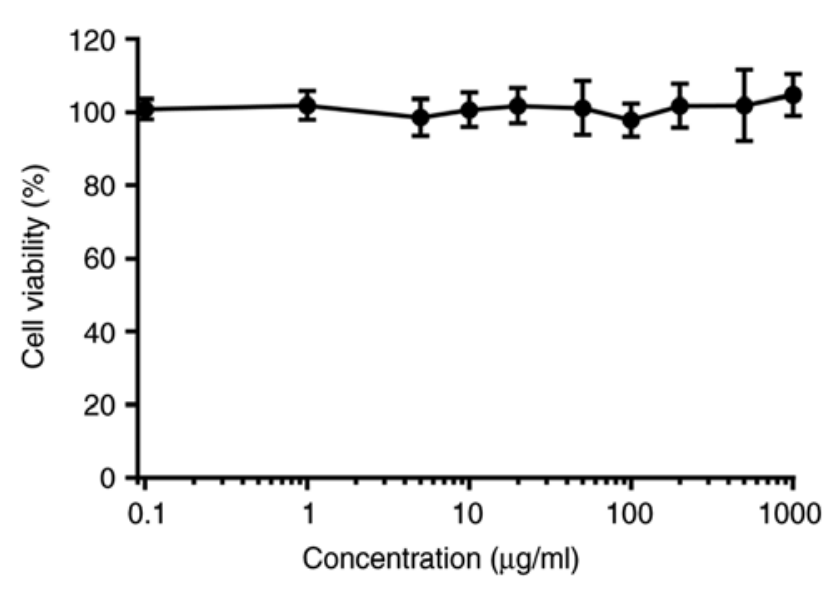

Figure 7. HT1080 cell viability following $48 \mathrm{~h}$ of treatment with poly ( $\beta$-malic acid).

PMLA has preferable biocompatibility and biodegradability and is non-toxic, therefore, it is considered a promising drug carrier material. In the present study, the antitumor drug DOX was attached to PMLA via a hydrazine bond to obtain a $\mathrm{pH}$-sensitive drug delivery conjugate. Drug release experiments at different $\mathrm{pH}$ conditions showed that the release of DOX was pH-dependent in PMLA-Hz-DOX, whereas the release behavior of PMLA-ami-DOX did not alter with the decrease of $\mathrm{pH}$. The in vitro biological experiments showed that grafting DOX onto PMLA reduced the toxicity of DOX. However, following uptake by cancer cells, DOX released from the PMLA-Hz-DOX conjugate due to the tumor-specific $\mathrm{pH}$ environment. PMLA-Hz-DOX, with its $\mathrm{pH}$-responding drug delivery properties, is expected to become a novel type of controlled release drug platform.

\section{Acknowledgements}

Not applicable.

\section{Funding}

The present study was partially funded by grants from the National Natural Science Foundation of China (grant nos. 81571786,31771087 and 31671015) and the Shaanxi Science \& Technology Co-ordination \& Innovation Project (grant no. 2015KTCL03-12). 

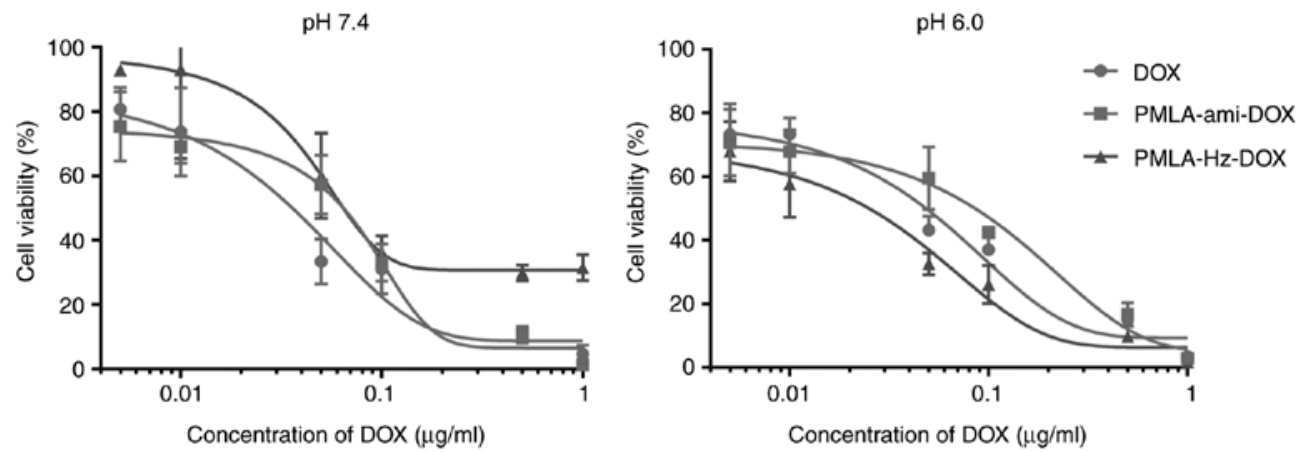

Figure 8. Viability of HT1080 cells following exposure to PMLA-Hz-DOX, PMLA-ami-DOX, and free DOX at various drug concentrations at $37^{\circ} \mathrm{C}$ for $48 \mathrm{~h}$. PMLA, poly ( $\beta$-malic acid); DOX, doxorubicin.
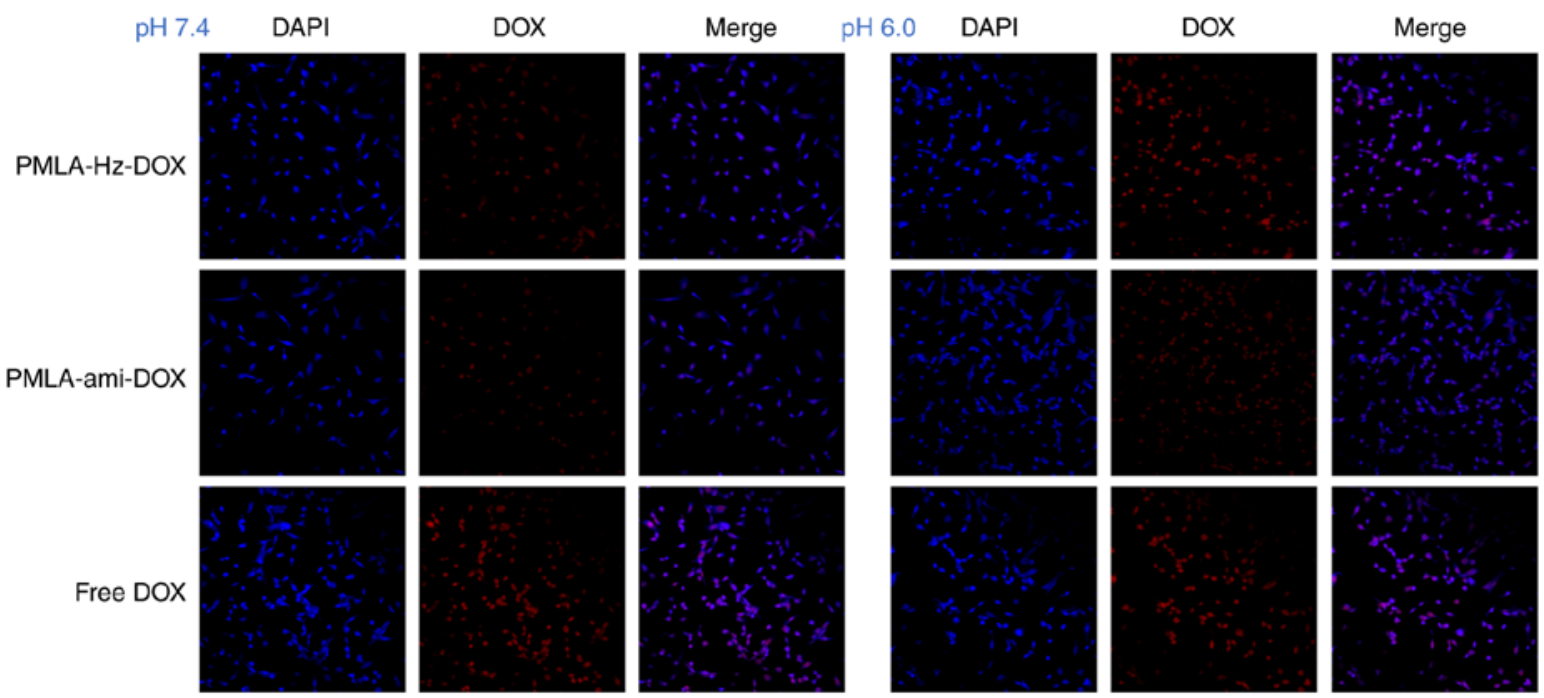

Figure 9. Internalization of different nanoconjugates in HT1080 cells at pH 7.4 and pH 6.8 imaged by confocal laser scanning microscopy at $2 \mathrm{~h}$. Magnification, x20. PMLA, poly ( $\beta$-malic acid); DOX, doxorubicin; DAPI, 4',6-diamidino-2-phenylindole.
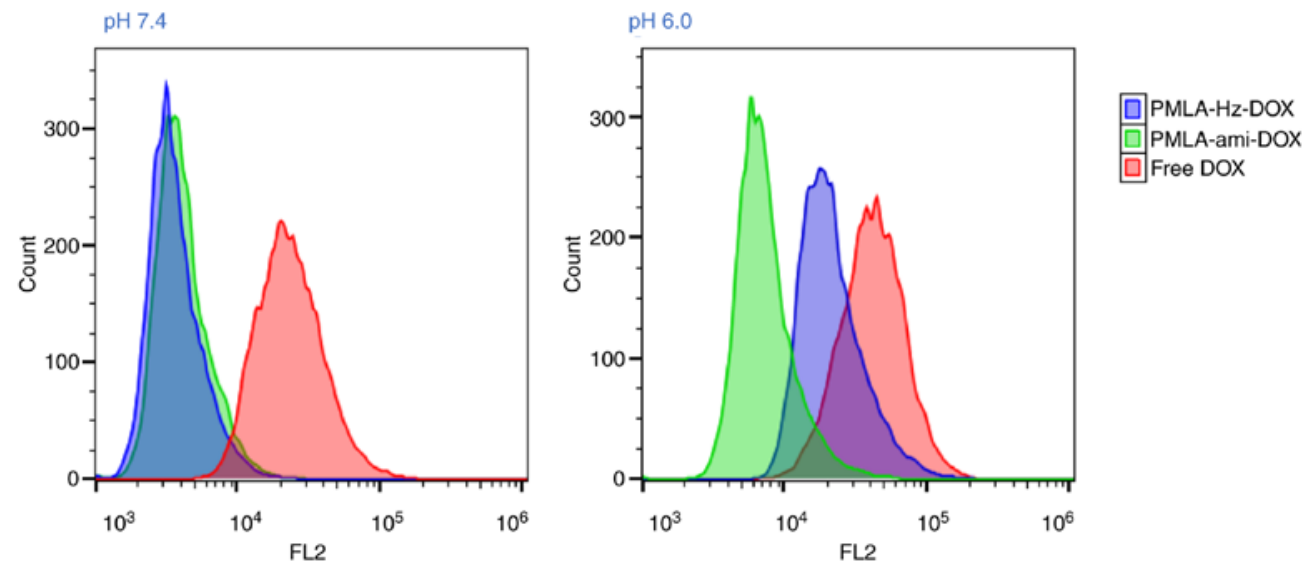

Figure 10. Cellular uptake of DOX-loaded nanoconjugates in HT1080 cells at pH 7.4 and pH 6.8, analyzed by flow cytometry at 2 h. PMLA, poly ( $\beta$-malic acid); DOX, doxorubicin.

\section{Availability of data and materials}

The datasets used or analysed during the current study are available from the corresponding author on reasonable request.

\section{Authors' contributions}

BL and YP performed the experiments and drafted the manuscript. EJ analyzed the data. YQ performed the experiments and analysed the data. HW made substantial contributions to 
the design of the present study and wrote the manuscript. All authors read and approved the final manuscript.

\section{Ethics approval and consent to participate}

Not applicable.

\section{Patient consent for publication}

Not applicable.

\section{Competing interests}

The authors confirm that they have no competing interests.

\section{References}

1. Kopecek J: Polymer-drug conjugates: Origins, progress to date and future directions. Adv Drug Deliv Rev 65: 49-59, 2013.

2. Larson $\mathrm{N}$ and Ghandehari H: Polymeric conjugates for drug delivery. Chem Mater 24: 840-853, 2012.

3. Li J, Yu F, Chen Y and Oupicky D: Polymeric drugs: Advances in the development of pharmacologically active polymers. J Contro Release 219: 369-382, 2015.

4. Khandare J and Minko T: Polymer-drug conjugates: Progress in polymeric prodrugs. Prog Polym Sci 31: 359-397, 2006.

5. Li C and Wallace S: Polymer-drug conjugates: Recent development in clinical oncology. Adv Drug Deliv Rev 60: 886-898, 2008

6. Feng Q and Tong R: Anticancer nanoparticulate polymer-drug conjugate. Bioeng Transl Med 1: 277-296, 2016.

7. Canal F, Sanchis J and Vicent MJ: Polymer-drug conjugates as nano-sized medicines. Curr Opin Biotechnol 22: 894-900, 2011.

8. Lee BS, Vert M and Holler E: Water-soluble aliphatic polyesters: Poly(malic acid)s. Wiley-VCH Verlag GmbH \& Co. KGaA, 2005

9. Braud C, Bunel $\mathrm{C}$ and Vert M: Poly( $\beta$-malic acid): A new polymeric drug-carrier. Polymer Bulletin 13: 293-299, 1985.

10. Loyer P and Cammas-Marion S: Natural and synthetic poly(malic acid)-based derivates: A family of versatile biopolymers for the design of drug nanocarriers. J Drug Target 22: 556-575, 2014.

11. Fernández CE, Mancera M, Holler E, Galbis JA and Muñoz-Guerra S: High molecular weight methyl ester of microbial poly( $\beta, 1-$ malic acid): Synthesis and crystallization. Polymer 47: 6501-6508, 2006.

12. Martinez Barbosa ME, Cammas S, Appel M and Ponchel G: Investigation of the degradation mechanisms of poly(malic acid) esters in vitro and their related cytotoxicities on J774 macrophages. Biomacromolecules 5: 137-143, 2004.

13. Segal E and Satchi-Fainaro R: Design and development of polymer conjugates as anti-angiogenic agents. Adv Drug Deliv Rev 61: 1159-1176, 2009.

14. Ljubimova JY, Portilla-Arias J, Patil R, Ding H, Inoue S, Markman JL, Rekechenetskiy A, Konda B, Gangalum PR, Chesnokova A, et al: Toxicity and efficacy evaluation of multiple targeted polymalic acid conjugates for triple-negative breast cancer treatment. J Drug Target 21: 956-967, 2013.

15. Lee BS, Fujita M, Khazenzon NM, Wawrowsky KA, Wachsmann-Hogiu S, Farkas DL, Black KL, Ljubimova JY and Holler E: Polycefin, a new prototype of a multifunctional nanoconjugate based on poly(beta-L-malic acid) for drug delivery. Bioconjug Chem 17: 317-326, 2006.

16. Ding H, Inoue $S$, Ljubimov AV, Patil R, Portilla-Arias J, Hu J, Konda B, Wawrowsky KA,Fujita M, Karabalin N, et al: Inhibition of brain tumor growth by intravenous poly (beta-L-malic acid) nanobioconjugate with $\mathrm{pH}$-dependent drug release [corrected]. Proc Natl Acad Sci USA 107: 18143-18148, 2010.
17. Qiao YB, Duan X, Fan L, Li W, Wu H and Wang YK: Synthesis of controlled molecular weight poly (beta-malic acid) and conjugation with HCPT as a polymeric drug carrier. J Polym Res 21: 397, 2014

18. Yang T,Li W, Duan X,Zhu L,Fan L, Qiao Y and Wu H: Preparation of two types of polymeric micelles based on poly(beta-L-malic acid) for antitumor drug delivery. PLoS One 11: e0162607, 2016.

19. Guo X, Shi C, Wang J, Di S and Zhou S: pH-triggered intracellular release from actively targeting polymer micelles. Biomaterials 34: 4544-4554, 2013.

20. Chen B, Dai W, He B, Zhang H, Wang X, Wang Y and Zhang Q: Current multistage drug delivery systems based on the tumor microenvironment. Theranostics 7: 538-558, 2017.

21. Huang X, Liao W, Zhang G, Kang S and Zhang CY: pH-sensitive micelles self-assembled from polymer brush (PAE-g-cholesterol)b-PEG-b-(PAE-g-cholesterol) for anticancer drug delivery and controlled release. Int J Nanomedicine 12: 2215-2226, 2017.

22. Wang X, Yang Y, Zhuang Y, Gao P, Yang F, Shen H, Guo H and $\mathrm{Wu} \mathrm{D}$ : Fabrication of $\mathrm{pH}$-responsive nanoparticles with an AIE feature for imaging intracellular drug delivery. Biomacromolecules 17: 2920-2929, 2016.

23. Zhao D, Xu JQ, Yi XQ, Zhang Q, Cheng SX, Zhuo RX and Li F: pH-activated targeting drug delivery system based on the selective binding of phenylboronic acid. ACS Appl Mater Interfaces 8: 14845-14854, 2016.

24. Sun T, Zhang YS, Pang B, Hyun DC, Yang M and Xia Y: Engineered nanoparticles for drug delivery in cancer therapy. Angew Chem Int Ed Engl 53: 12320-12364, 2014.

25. Karimi M, Eslami M, Sahandi-Zangabad P, Mirab F, Farajisafiloo N,ShafaeiZ, GhoshD,Bozorgomid M,DashkhanehF and Hamblin MR: pH-sensitive stimulus-responsive nanocarriers for targeted delivery of therapeutic agents. Wiley Interdiscip Rev Nanomed Nanobiotechnol 8: 696-716, 2016.

26. Qian CG, Chen YL, Feng PJ, Xiao XZ, Dong M, Yu JC, Hu QY, Shen QD and Gu Z: Conjugated polymer nanomaterials for theranostics. Acta Pharmacol Sin 38: 764-781, 2017.

27. Zhu YJ and Chen F: pH-responsive drug-delivery systems. Chem Asian J 10: 284-305, 2015.

28. Wang Z, Deng X, Ding J, Zhou W, Zheng X and Tang G: Mechanisms of drug release in $\mathrm{pH}$-sensitive micelles for tumour targeted drug delivery system: A review. Int J Pharm 535: 253-260, 2018 .

29. Lee ES, Oh KT, Kim D, Youn YS and Bae YH: Tumor $\mathrm{pH}$-responsive flower-like micelles of poly(L-lactic acid)-b-poly(ethylene glycol)-b-poly(L-histidine). J Control Release 123: 19-26, 2007.

30. Zhang H, Li F, Yi J, Gu C, Fan L, Qiao Y, Tao Y, Cheng C and $\mathrm{Wu} \mathrm{H}$ : Folate-decorated maleilated pullulan-doxorubicin conjugate for active tumor-targeted drug delivery. Eur J Pharm Sci 42: 517-526, 2011.

31. National Pharmacopoeia Committee: Pharmacopoeia of People's Republic of China. China Medical Science Press, Beijing. Part 2: 955,2015

32. Zhou Q, Hou Y, Zhang L, Wang J, Qiao Y, Guo S, Fan L, Yang T, Zhu L and Wu H: Dual-pH sensitive charge-reversal nanocomplex for tumor-targeted drug delivery with enhanced anticancer activity. Theranostics 7: 1806-1819, 2017.

33. Mintzer MA and Simanek EE: Nonviral vectors for gene delivery. Chem Rev 109: 259-302, 2009.

34. Aoshima Y, Hokama R, Sou K, Sarker SR, Iida K, Nakamura H, Inoue $\mathrm{T}$ and Takeoka $\mathrm{S}$ : Cationic amino acid based lipids as effective nonviral Gene delivery vectors for primary cultured neurons. ACS Chem Neurosci 4: 1514-1519, 2013.

35. Zhao G, Long L, Zhang L, Peng M, Cui T, Wen X, Zhou X, Sun L and Che L: Smart pH-sensitive nanoassemblies with cleavable PEGylation for tumor targeted drug delivery. Sci Rep 7: 3383 , 2017.

36. Liu Y, Li D, Guo X, Xu H, Li Z, Zhang Y, Song C, Fan R, Tang $\mathrm{X}$ and $\mathrm{Zhang} \mathrm{Z}$ : A pH-responsive prodrug delivery system of 10-HCPT for controlled release and tumor targeting. Int J Nanomedicine 12: 2227-2242, 2017. 